\title{
Avances biotecnológicos sobre maricultura en Costa Rica. Una revisión de la investigación desarrollada por la Escuela de Ciencias Biológicas de la Universidad Nacional
}

\author{
Biotechnological Advances on Mariculture in Costa Rica. A Review of the \\ Investigation Developed by the School of Biological Sciences of the National University
}

\author{
Rebeca Rojas-Alfaro \\ rebeca.rojas.alfaro@una.cr
}

Estación de Biología Marina

Escuela de Ciencias Biológicas, Universidad Nacional

Heredia, Costa Rica

Carolina Sancho-Blanco

carolina.sancho.blanco@una.cr

Laboratorio de Análisis Genómico

Escuela de Ciencias Biológicas, Universidad Nacional

Heredia, Costa Rica

Luis Vega-Corrales

luis.vega.corrales@una.cr

Estación de Biología Marina

Escuela de Ciencias Biológicas, Universidad Nacional

Heredia, Costa Rica

Recibido-Received: 13/dic/2015 / Corregido-Corrected: 5/set /2016.

Aceptado-Accepted: 23/set/2016 / Publicado-Published: 31/jul /2017.

\begin{abstract}
Resumen
El desarrollo de la maricultura en Costa Rica ha requerido de la validación, la innovación y la generación de conocimiento científico biotecnológico. Se presenta una revisión de los principales avances biotecnológicos sobre maricultura que han sido aplicados y desarrollados por la Escuela de Ciencias Biológicas de la Universidad Nacional de Costa Rica. La experimentación y optimización de la tecnología del cultivo de Crassostrea gigas ha impulsado la ostricultura en el país y el surgimiento de emprendimientos locales. La investigación científica realizada ha generado avances biotecnológicos de relevancia mundial sobre la biología reproductiva de camarones peneidos y ha permitido la reproducción controlada de peces marinos de interés comercial del Golfo de Nicoya, Costa Rica. Además, se han aplicado y evaluado técnicas biotecnológicas para el cultivo de microalgas, depuración bacteriana de moluscos, vigilancia de Floraciones Algales Nocivas y biorremediación. Se debe fortalecer
\end{abstract}


UNICIENCIA Vol. 31, No. 2, pp. 111-119. Julio-diciembre, 2017.

ISSN Electrónico: 2215-3470

URL: www.revistas.una.ac.cr/uniciencia

DOI: $\underline{\text { http://dx.doi.org/10.15359/ru.31-2.8 }}$

Email: revistauniciencia@una.cr

la investigación biotecnológica sobre maricultura, fomentar la transferencia de la biotecnología desarrollada y promover la protección por medio de la propiedad intelectual de la innovación biotecnológica generada como herramientas para el desarrollo económico y social en Costa Rica.

Palabras claves: Biotecnología; acuicultura marina; Costa Rica.

\begin{abstract}
The development of mariculture in Costa Rica has required validation, innovation and generation of scientific-biotechnological knowledge. A revision of the main biotechnological advances on mariculture that have been applied and developed by the School of Biological Sciences of the National University of Costa Rica is presented. Experimentation and optimization of culture technology have boosted Crassostrea gigas oyster farming in the country and the establishment of local enterprises. Scientific research has generated globally significant biotechnological advances on the reproductive biology of penaeid shrimp and has allowed the controlled reproduction of commercial marine fish in the Gulf of Nicoya, Costa Rica. In addition, biotechnological techniques have been implemented and evaluated for growing algae, bacterial depuration of molluscs, Harmful Algal Blooms monitoring and bioremediation. Biotechnology research on mariculture and the transfer of new biotechnologies must be promoted and protected by the Intellectual Property of biotechnological innovations as tools for economic and social development in Costa Rica.
\end{abstract}

Keywords: biotechnology; marine aquaculture; Costa Rica.

El desarrollo de la maricultura en Costa Rica ha implicado la transferencia de tecnologías de cultivo, validación de metodologías, experimentación e investigación para el cultivo de especies nativas e introducidas. La innovación y la generación de conocimiento científico biotecnológico sobre maricultura en Costa Rica ha sido un área de investigación de la Escuela de Ciencias Biológicas de la Universidad Nacional (ECB-UNA). En la Estación de Biología Marina Juan Bertoglia Richards de la ECB-UNA se ubican los laboratorios de investigación sobre reproducción y cultivo de peces, moluscos, crustáceos y microalgas, así como el Laboratorio de Microbiología Marina, Fitoplancton Marino, entre otros. Estos laboratorios han aplicado y desarrollado avances biotecnológicos sobre cultivo, reproducción, inmunología, biología molecular, biorremediación, depuración e inocuidad, con el fin de fomentar la maricultura en el país.

Actualmente, la biotecnología y la maricultura son áreas de investigación atinentes con el Plan Nacional de Ciencia, Tecnología e Innovación 2015-2020. Uno de los principales retos en Costa Rica es el desarrollo de buenas prácticas productivas basadas en la ciencia y tecnología para mejorar las oportunidades de los sectores agrícolas y de pesca (MICITT, 2015). A nivel mundial, la maricultura ha crecido aceleradamente y se ha consolidado como una alternativa para la producción de organismos acuáticos que contribuye con la seguridad alimentaria y con el desarrollo socioeconómico de las comunidades costeras de países en desarrollo (FAO, 2014). De esta manera, la biotecnología y la maricultura son áreas de investigación de la ECB-UNA, fundamentales para el desarrollo de Costa Rica.

Se presenta una revisión de los principales avances biotecnológicos sobre maricultura, que han sido aplicados y desarrollados por la Escuela de Ciencias Biológicas de la Universidad Nacional de Costa Rica. 
ISSN Electrónico: 2215-3470

DOI: http://dx.doi.org/10.15359/ru.31-2.8
UNICIENCIA Vol. 31, No. 2, pp. 111-119. Julio-diciembre, 2017.

URL: www.revistas.una.ac.cr/uniciencia Email: revistauniciencia@una.cr

\section{Líneas de investigación desarrolladas}

\section{- Moluscos}

Los avances biotecnológicos aplicados y desarrollados en el Laboratorio de Reproducción y Cultivo de Moluscos han validado y optimizado la tecnología del cultivo de la ostra Crassostrea gigas en Costa Rica. En la década de los noventa, la ECB-UNA realizó los primeros ensayos para la producción de semillas y el cultivo de ostras en el país (Arias, 2000; Zúñiga, 2002). El trabajo de extensión realizado ha permitido la transferencia de esta tecnología a pequeños grupos organizados, impulsando la ostricultura en el país y el surgimiento de emprendimientos locales.

Esta línea de investigación-extensión ha promovido el trabajo interdisciplinario, multidisciplinario e interinstitucional. La biotecnología aplicada en el Laboratorio de Cultivo de Plancton ha sido fundamental para la investigación sobre ostricultura y reproducción de peces y crustáceos. Este laboratorio ha optimizado la tecnología para el cultivo de microalgas referentes para la maricultura y ha aislado especies endógenas del Golfo de Nicoya. Rodríguez (2014) evaluó la factibilidad de cultivo y composición bioquímica de Nitzschia sp. y Chaetoceros sp. aisladas del Golfo de Nicoya. Estas cepas presentaron alto potencial biotecnológico, debido a que son aptas para el cultivo en condiciones controladas y, de acuerdo con el tamaño de la célula, tasa de crecimiento, productividad y composición bioquímica, podrían ser utilizadas como alimento en la maricultura de especies tropicales.

El Laboratorio de Microbiología Marina evaluó un sistema, diseñado por el Laboratorio de Cultivo y Reproducción de Moluscos, para la depuración bacteriana de las ostras cultivadas en el Golfo de Nicoya. Los resultados confirmaron la efectividad del diseño del sistema para la depuración de indicadores bacterianos de contaminación fecal en ostras. Esta tecnología ofrece una alternativa de bajo costo para la eliminación poscosecha de contaminantes bacterianos en las ostras, genera valor agregado y reduce los riesgos potenciales a la salud pública que podría ocasionar el consumo de estos moluscos (Vega, Marín, Pacheco \& Zúñiga, 2013). Además, este laboratorio evaluó, utilizando Escherichia coli como bioindicador universal de contaminación fecal, la calidad sanitaria de las zonas de cultivo de ostras de Punta Cuchillo y Punta Morales en el Golfo de Nicoya (Vega \& Marín, 2014).

Por otra parte, un representante del Laboratorio de Fitoplancton Marino es integrante del Órgano Asesor de Consulta y Coordinación para la Vigilancia Epidemiológica de la Marea Roja en Costa Rica (Diario Oficial La Gaceta, 2016). La vigilancia de las floraciones algales nocivas (FAN) que realiza este laboratorio en el Golfo de Nicoya ha sido de gran importancia para el avance de la ostricultura en el país. Este laboratorio es de referencia nacional y ha logrado, mediante técnicas biotecnológicas, aislar y cultivar cepas de fitoplancton potencialmente tóxicas y formadoras de FAN del Golfo de Nicoya. Se han realizado investigaciones para la detección de toxinas de tipo paralizante por cromatografía de alta eficiencia en estas especies (Berrocal, 2011). De esta manera, la biotecnología aplicada para la depuración bacteriana de moluscos y para la evaluación sanitaria de las zonas de cultivo de ostras en el Golfo de Nicoya, así como la vigilancia de las FAN, han fortalecido la ostricultura en el país y demuestran la responsabilidad social que ha ejercido la ECB-UNA en el tema de maricultura y salud pública. 
UNICIENCIA Vol. 31, No. 2, pp. 111-119. Julio-diciembre, 2017.

ISSN Electrónico: 2215-3470

URL: www.revistas.una.ac.cr/uniciencia

DOI: http://dx.doi.org/10.15359/ru.31-2.8

Email: revistauniciencia@una.cr

\section{- Crustáceos}

La investigación científica desarrollada en el Laboratorio de Fisiología Reproductiva de Crustáceos ha generado avances biotecnológicos de relevancia mundial sobre la biología reproductiva de camarones peneidos. Se definió que la maduración sexual en machos de Litopenaeus stylirostris es controlada en tres niveles independientes: maduración de los testículos, maduración de los vasos deferentes y síntesis del espermatóforo (시aro, 1993). Se demostró que la ablación unilateral del pedúnculo ocular mejora la calidad de los espermatóforos en $L$. vannamei (Alfaro \& Lozano, 1993). Se evaluó el efecto de la infección bacteriana causada por Vibrio alginolyticus y Pseudomonas putrefaciens sobre la melanización del sistema reproductor y el deterioro de los espermatóforos en L. setiferus, dos de los principales problemas que afectan la sexualidad masculina en los peneidos (Alfaro, Lawrence \& Lewis, 1993). Además, se evaluó el comportamiento reproductivo de hembras de L. stylirostris respecto a $V$. alginolyticus, patógeno bacteriano que afecta los sistemas de maduración de reproductores de camarones marinos en todo el mundo (Pizarro \& Alfaro, 1994). Por otra parte, se reportó que la inyección de $17 \alpha$-metiltestosterona en L. vannamei mejora la calidad de los espermatóforos, en términos de que incrementa el recuento espermático y el peso del espermatóforo y reduce la incidencia de anormalidades en los espermatozoides (Alfaro, 1996).

En este laboratorio se han diseñado técnicas biotecnológicas para demostrar procesos fisiológicos sobre biología reproductiva de camarones peneidos. Se aplicó una técnica biotecnológica para evaluar la inducción in vitro de la activación espermática en Trachypenaeus byrdi, Xiphopenaeus riveti y L. occidentalis del Golfo de Nicoya. La técnica demostró que los espermatozoides requieren ser madurados en los receptáculos seminales de T. byrdi y $X$. riveti y en la superficie externa del télico de L. occidentalis (Alfaro, Muñoz, Vargas \& Komen, 2003). Además, se desarrolló una técnica eficiente para la inducción de la maduración ovárica y el desove en L. stylirostris y L. vannamei como alternativa a la tradicional y destructiva técnica de ablación del pedúnculo ocular. Los autores utilizaron la combinación de un neurotransmisor (serotonina) y una dopamina antagonista (espiperona), y obtuvieron porcentajes de maduración y desove similares a los obtenidos con la técnica de ablación del pedúnculo ocular, lo cual la convierte en una técnica de relevancia mundial para la inducción ovárica en camarones peneidos (Alfaro, Zúñiga \& Komen, 2004).

Otro aporte científico fue el desarrollo de investigaciones que establecieron la base para la manipulación in vitro de la activación de ovocitos en L. occidentalis, L. stylirostris y L. vannamei. Se describió la sincronización y la secuencia de los eventos asociados con la activación de los ovocitos y se evaluó el efecto de la temperatura, la concentración de $\mathrm{Mg}^{2+}$ y la disección ovárica durante este proceso. Además, se realizó un ensayo in vitro para evaluar la unión primaria interespecífica e intraespecífica entre las especies de Litopenaeus. El modelo biotecnológico desarrollado permitió una mejor comprensión del mecanismo de fertilización en camarones de télico abierto ( malla filamentosa en el espermatozoide de L. occidentalis, L. stylirostris y L. vannamei. La síntesis de esta región resultó ser diferente entre las especies estudiadas. Los autores concluyeron que la maduración acrosómica depende de la síntesis total de la malla filamentosa. Además, determinaron que después del apareamiento, las células espermáticas maduras de L. occidentalis sufren una serie de cambios fisiológicos que le otorgan la capacidad de reaccionar con los ovocitos presentes en el agua (Alfaro, Ulate \& Vargas, 2007). 
Seguidamente, se evaluó el efecto de la hormona juvenil III y del farnesoato de metilo sobre la calidad de espermatóforos en L. vannamei. El tratamiento con farnesoato de metilo causó un incremento significativo en número de espermatozoides y una disminución en anormalidades de estos mismos (Alfaro, Zúñiga, García \& Rojas, 2008). Posteriormente, se desarrolló una técnica para evaluar en L. vannamei la respuesta de reconocimiento contra implantes de ganglios torácicos de la misma especie y de L. stylirostris, T. byrdi y Macrobrachium tenellum. Los implantes interespecíficos e intraespecíficos no mantuvieron su integridad dentro de la cavidad corporal de L. vannamei (Alfaro, Hernández, Zúñiga, Soto \& Mejía-Arana, 2009). A partir de este estudio, se evaluó el uso de implantes y extractos de ganglios torácicos de hembras maduras de T. byrdi, $X$. riveti y $L$. occidentalis como inductor potencial para la maduración sexual de $L$. stylirostris, $L$. vannamei y T. byrdi. La técnica biotecnológica desarrollada no fue capaz de inducir una respuesta clara en la maduración gonadal de camarones peneidos (Alfaro \& Vega, 2010).

Además, se desarrolló el primer estudio sobre hibridación entre L. occidentalis, L. stylirostris y L. vannamei. Las inseminaciones artificiales intraespecíficas generaron desoves viables con un $54.2 \%$ de embriones normales, mientras que las inseminaciones artificiales interespecíficas generaron desoves infértiles con un alto porcentaje de huevos con corpúsculos intracelulares anormales. De esta manera, los autores demostraron la incompatibilidad esperma-ovocito en las inseminaciones artificiales interespecíficas (Ulate \& Alfaro-Montoya, 2010).

Otro descubrimiento realizado fue la ubicación y descripción de la estructura completa de la glándula androgénica de L. setiferus, L. occidentalis, L. stylirostris y Rimapenaeus byrdi (anteriormente Trachypenaeus byrdi). Esta investigación mejoró el conocimiento de la organización general del sistema reproductivo de machos de Litopenaeus y Rimapenaeus y aportó una base anatómica para la manipulación de la glándula androgénica (Alfaro-Montoya \& Hernández, 2012). Las primeras observaciones de la ultraestructura de la glándula androgénica en camarones peneidos fueron realizadas por Alfaro-Montoya (1994). Recientemente, se evaluó el potencial de la ablación de la glándula androgénica para la reversión sexual en L. vannamei como alternativa biotecnológica para la producción de cultivos monosexuales de hembras. Los machos a los que se les removió la glándula androgénica presentaron reducción en el crecimiento, regeneración de caracteres sexuales, espermatogénesis activa y degradación de espermátides y tejidos reproductivos, por lo tanto, no fue posible una reversión sexual total (Alfaro-Montoya, Hernández-Noguera, Vega-Alpízar \& Umaña-Castro, 2015).

Además, se evaluó el efecto del ibuprofeno en la biosíntesis de prostaglandinas y en la reproducción de machos y hembras de L. occidentalis, L. stylirostris y L. vannamei. Se obtuvo una mayor tasa de hembras de L. vannamei con ovarios en desarrollo. En L. stylirostris no se activó la vitelogénesis. En los machos, el ibuprofeno no ejerció ningún efecto sobre el recuento espermático y el peso del espermatóforo, sin embargo, redujo el número de anomalías espermáticas en L. occidentalis y L. stylirostris y mejoró la renovación del espermatóforo en $L$. stylirostris. Los hallazgos de esta investigación apoyan la hipótesis de que las prostaglandinas están implicadas en la reproducción de peneidos (Alfaro-Montoya, 2015).

Actualmente, el Laboratorio de Fisiología Reproductiva de Crustáceos en conjunto con el Laboratorio de Análisis Genómico (LAGEN) de la ECB-UNA y la cooperación de la Universidad Ben-Gurión del Néguev, Israel, se encuentran desarrollando investigaciones para la reversión sexual en peneidos. Se ha realizado la identificación molecular de transcriptos codificantes del 
péptido tipo insulina de la glándula androgénica en L. occidentalis, L. stylirostris y L. vannamei. La identificación y caracterización de las secuencias de la glándula androgénica involucradas en los procesos reproductivos permitirá la reversión sexual mediante silenciamiento génico por ARN interferente. El desarrollo de la manipulación biotecnológica del sexo permitirá obtener poblaciones monosexuales de hembras y reducir el efecto del dimorfismo sexual en el crecimiento en camarones peneidos ( $\underline{\text { Sancho, 2015). }}$.

Por otra parte, en la Estación de Biología Marina se están aplicando y optimizando técnicas biotecnológicas para la biorremediación de agua en sistemas de cultivo recirculados. Se demostró que el flujo de agua ejerce un efecto importante en la capacidad de los tapetes microbianos para la biorremediación de amonio y nitritos en un sistema de recirculación piloto para el cultivo de L. vannamei (ذiménez-Montealegre, Zamora-Castro \& Zúñiga-Calero, 2015). Se definió que la efectividad de remoción de nitrógeno inorgánico disuelto en un sistema recirculado de cultivo de L. vannamei no depende, según el diseño del estudio, de la densidad de colonización de los tapetes microbianos (

\section{- Peces}

En el Laboratorio de Cultivo y Reproducción de Peces Marinos se han desarrollado investigaciones biotecnológicas sobre reproducción controlada de especies de interés comercial del Golfo de Nicoya. Se evaluó el uso del extracto de pituitaria de carpa en la maduración y desove de hembras silvestres de pargo manchado Lutjanus guttatus (Valverde, 1999; Valverde y Boza, 1998) y el efecto de la hormona gonadotropina coriónica humana $(\mathrm{GCH})$ y del extracto de pituitaria de $L$. guttatus en la maduración in vitro de ovocitos de esta especie (López, 2004). Las técnicas aplicadas permitieron lograr desoves naturales y desoves inducidos utilizando la hormona GCH en hembras maduras de L. guttatus (Boza-Abarca, Calvo-Vargas, Solís-Ortiz \& Komen, 2008). Posteriormente, se evaluó la inducción hormonal en peces silvestres y criados en jaulas flotantes de L. guttatus utilizando una suspensión de pituitaria de carpa y GCH. Este estudio logró optimizar la técnica con GCH y generar mayor cantidad de huevos desovados y mejores porcentajes de fertilización (Boza-Abarca, Valverde-Chavarría, Calvo-Vargas \& Ramírez-Alvarado, 2011).

Recientemente, se describió por primera vez la reproducción en cautiverio, el desarrollo embrionario y larval y el crecimiento de juveniles de la corvina aguada Cynoscion squamipinnis (Barquero, 2015; Boza-Abarca, Ramírez-Alvarado, Barquero-Chanto, Calvo-Vargas \& BerrocalArtavia, 2016a, 2016b).

\section{Perspectivas}

La ECB-UNA debe potenciar la investigación e innovación biotecnológica sobre maricultura. Debe fortalecer el trabajo de extensión desarrollado por el Laboratorio de Cultivo y Reproducción de Moluscos y fomentar la transferencia de la biotecnología desarrollada por las otras líneas de investigación. Es importante promover la investigación y la vigilancia realizada en el tema de maricultura y salud pública. Es necesario impulsar líneas de investigación sobre patologías y sanidad acuícola e implementar programas de mejoramiento genético. Además, debe incentivar la investigación dirigida al mejoramiento de la reproducción controlada de organismos marinos mediante técnicas de biología molecular. Se debe promover la protección por medio de la propiedad intelectual de la innovación biotecnológica generada sobre maricultura como herramienta para el desarrollo económico y social en Costa Rica. 
ISSN Electrónico: 2215-3470

DOI: http://dx.doi.org/10.15359/ru.31-2.8
UNICIENCIA Vol. 31, No. 2, pp. 111-119. Julio-diciembre, 2017. URL: www.revistas.una.ac.cr/uniciencia Email: revistauniciencia@una.cr

\section{Agradecimientos}

Al Ph.D. Jorge Alfaro Montoya por la revisión del manuscrito.

\section{Referencias}

Alfaro, J. (1993). Reproductive quality evaluation of male Penaeus stylirostris from a grow-out pond. Journal of the World Aquaculture Society, 24(1), 6-11. http://dx.doi.org/10.1111/j.1749-7345.1993. $\underline{\mathrm{tb} 00144 . \mathrm{x}}$

Alfaro, J. (1996). Effect of 17a-methyltestosterone and 17a-hydroxyprogesterone on the quality of white shrimp Penaeus vannamei spermatophores. Journal of the World Aquaculture Society, 27(4), 487492. http://dx.doi.org/10.1111/j.1749-7345.1996.tb00634.x

Alfaro, J., \& Lozano, X. (1993). Development and deterioration of spermatophores in pond-reared Penaeus vannamei. Journal of the World Aquaculture Society, 24(4), 522-529. http://dx.doi. org/10.1111/j.1749-7345.1993.tb00583.x

Alfaro, J., \& Vega, L. (2010). Effects of transplants and extracts of thoracic nerve cord-ganglia on gonad maturation of penaeoid shrimp. Aquaculture Research, 41(2), 182-188. http://dx.doi. org/10.1111/j.1365-2109.2009.02315.x

Alfaro, J., Hernández, L., Zúñiga, G., Soto, R., \& Mejía-Arana, F. (2009). Thoracic nerve cord-ganglia recognition in intraspecific and interspecific transplants in the white shrimp, Penaeus (Litopenaeus) vannamei. Aquaculture, 288(1), 126-131. http://dx.doi.org/10.1016/j.aquaculture.2008.11.006

Alfaro, J., Lawrence, A., \& Lewis, D. (1993). Interaction of bacteria and male reproductive system blackening disease of captive Penaeus setiferus. Aquaculture 117(1), 1- 8. http://dx.doi. org/10.1016/0044-8486(93)90117-H

Alfaro, J., Muñoz, N., Vargas, M., \& Komen, J. (2003). Induction of sperm activation in open and closed thelycum penaeoid shrimps. Aquaculture, 216(1), 371-381. http://dx.doi.org/10.1016/ $\underline{\text { S0044-8486(02)00514-8 }}$

Alfaro, J., Ulate, K., \& Vargas, M. (2007). Sperm maturation and capacitation in the open thelycum shrimp Litopenaeus (Crustacea: Decapoda: Penaeoidea). Aquaculture, 270(1), 436-442. http:// dx.doi.org/10.1016/j.aquaculture.2007.05.011

Alfaro, J., Zúñiga, G., \& Komen, J. (2004). Induction of ovarian maturation and spawning by combined treatment of serotonin and a dopamineantagonist, spiperone in Litopenaeusstylirostrisand Litopenaeus vannamei. Aquaculture, 236(1), 511-522. http://dx.doi.org/10.1016/j.aquaculture.2003.09.020

Alfaro, J., Zúñiga, G., García, A., \& Rojas, E. (2008). Preliminary evaluation of the effect of juvenile hormone III and methyl farnesoate on spermatophore quality of the white shrimp, Litopenaeus vannamei Boone, 1931 (Decapoda: Penaeidae). Revista de Biología Marina y Oceanografía, 43(1), 167-171.http://dx.doi.org/10.4067/S0718-19572008000100018

Alfaro-Montoya, J. (1994). Ultraestructura de la glándula androgénica, espermatogénesis y oogénesis de camarones marinos (Decapoda: Penaeidae). Revista de Biología Tropical, 42(Supl 2), 121-129.

Alfaro-Montoya, J. (2015). The effect of Ibuprofen on female and male reproduction of the open thelyca marine shrimp, Litopenaeus. Aquaculture Research, 46(1), 105-116. http://dx.doi.org/10.1111/ $\underline{\operatorname{are} .12163}$

Alfaro-Montoya, J., \& Hernández, L. (2012). The histological structure of the androgenic gland and cellular cord of the male reproductive system of adult Litopenaeus and Rimapenaeus byrdi. Journal of Crustacean Biology, 32(3), 351-357. http://dx.doi.org/10.1163/193724012X626511 
UNICIENCIA Vol. 31, No. 2, pp. 111-119. Julio-diciembre, 2017.

ISSN Electrónico: 2215-3470

URL: www.revistas.una.ac.cr/uniciencia

DOI: http://dx.doi.org/10.15359/ru.31-2.8

Email: revistauniciencia@una.cr

Alfaro-Montoya, J., Hernández-Noguera, L., Vega-Alpízar, L., \& Umaña-Castro, R. (2015). Effects of androgenic gland ablation on growth, sexual characters and spermatogenesis of the white shrimp, Litopenaeus vannamei (Decapoda: Penaeidae) males. Aquaculture Research (aceptado para publicación). http://dx.doi.org/10.1111/are.12727

Arias, S. (2000). Estudio técnico para la producción de semilla de ostra (Crassostrea rhizophorae y Crassostrea gigas) en condiciones de laboratorio (Tesis de licenciatura inédita). Universidad Nacional, Heredia, Costa Rica.

Barquero, J. (2015). Descripción del desarrollo ontogénico y el crecimiento de la corvina aguada, Cynoscion squamipinnis (Sciaenidae) para la optimización de su cultivo (Tesis de licenciatura inédita). Universidad Nacional, Heredia, Costa Rica.

Berrocal, K. (2011). Perfil de toxinas de tipo paralizante de algunas especies de dinoflagelados del Golfo de Nicoya, Puntarenas, Costa Rica (Tesis de licenciatura inédita). Universidad Nacional, Heredia, Costa Rica.

Boza-Abarca, J., Calvo-Vargas, E., Solís-Ortiz, N., \& Komen, J. (2008). Induced spawning and larval rearing of spotted rose snapper, Lutjanus guttatus, at the Marine Biology Station, Puntarenas, Costa Rica. Ciencias Marinas, 34(2), 239-252.

Boza-Abarca, J., Ramírez-Alvarado, M., Barquero-Chanto, J., Calvo-Vargas, E., \& Berrocal-Artavia, K. (2016a). Crecimiento de juveniles de la corvina aguada, Cynoscion squamipinnis (Perciformes: Scianidae) en cautiverio. Uniciencia, 30(2), 63-74. http://dx.doi.org/10.15359/ru.30-2.5

Boza-Abarca, J., Ramírez-Alvarado, M., Barquero-Chanto, J., Calvo-Vargas, E., \& Berrocal-Artavia, K. (2016b). Desove espontáneo, ontogenia y crecimiento en cautiverio de Cynoscion squamipinnis (Perciformes: Scianidae). Revista de Biología Tropical (aceptado para publicación).

Boza-Abarca, J., Valverde-Chavarría, S., Calvo-Vargas, E., Ramírez-Alvarado, M., \& Rodríguez-Gómez, E. (2011). Hormone-induced spawning of wild and captive-grown spotted rose snapper Lutjanus guttatus using carp pituitary suspension and human chorionic gonadotropin. Ciencias Marinas, 37(2), 125-139. http://dx.doi.org/10.7773/cm.v37i2.1802

Diario Oficial La Gaceta. (2016). Decreto Ejecutivo N³9669-MAG-S, Comisión para la Vigilancia Epidemiológica de la Marea Roja. San José, Costa Rica: Imprenta Nacional.

FAO. Organización de las Naciones Unidas para la Alimentación y la Agricultura (2014). El estado mundial de la pesca y la acuicultura 2014. Roma, Italia: Autor.

Jiménez-Montealegre, R., Zamora-Castro, J., \& Zúñiga-Calero, G. (2015). Determinación del flujo de agua para la biorremediación en sistemas recirculados acuaculturales utilizando tapetes microbianos construidos. Latin American Journal of Aquatic Research, 43(1), 234-247. http://dx.doi.org/10.3856/ vol43-issue1-fulltext-20

López, A. (2004). Determinación in vitro de la potencia del extracto de pituitaria del pargo mancha (Lutjanus guttatus) (Steindachner, 1869) (Tesis de licenciatura inédita). Universidad Nacional, Heredia, Costa Rica.

MICITT. Ministerio de Ciencia, Tecnología y Telecomunicaciones (2015). Plan Nacional Ciencia, Tecnología e Innovación 2015-2021. San José, Costa Rica: Autor.

Pizarro, F., \& Alfaro, J. (1994). Reproductive performance of Penaeus stylirostris females injected with heat-killed Vibrio alginolyticus. Journal of the Word Aquaculture Society, 25(4), 576-579. http:// dx.doi.org/10.1111/j.1749-7345.1994.tb00828.x

Ramírez, S. (2015). Biorremediación de nitrógeno disuelto en un sistema recirculado de agua marina utilizando tapetes microbianos (Tesis de licenciatura inédita). Universidad Nacional, Heredia, Costa Rica. 
ISSN Electrónico: 2215-3470

DOI: http://dx.doi.org/10.15359/ru.31-2.8
UNICIENCIA Vol. 31, No. 2, pp. 111-119. Julio-diciembre, 2017. URL: www.revistas.una.ac.cr/uniciencia Email: revistauniciencia@una.cr

Rodríguez, K. (2014). Aislamiento, factibilidad del cultivo y composición bioquímica de dos géneros de diatomeas de la costa del Pacífico de Costa Rica (Tesis de maestría inédita). Universidad Católica del Norte, Coquimbo, Chile.

Rojas, E., \& Alfaro, J. (2007). In vitro manipulation of egg activation in the open thelycum shrimp Litopenaeus. Aquaculture, 264(1), 469-474. http://dx.doi.org/10.1016/j.aquaculture.2006.12.025

Sancho, C. (2015). Aislamiento y caracterización de la secuencia parcial del transcripto codificante tipo insulina de la glándula androgénica en camarones marinos del género Litopenaeus (Tesis de licenciatura inédita). Universidad Nacional, Heredia, Costa Rica.

Ulate, K., \& Alfaro-Montoya, J. (2010). Sperm-egg incompatibility in inter-specific inseminations of Penaeus (Litopenaeus) occidentalis, P. (Litopenaeus) stylirostris and P. (Litopenaeus) vannamei. Aquaculture, 309(1), 290-292. http://dx.doi.org/10.1016/j.aquaculture.2010.08.029

Valverde, S. (1999). Determinación de la dosis hormonal que estimula la maduración final y el desove en hembras de pargo mancha (Lutjanus guttatus, Steindachner 1869) (Tesis de licenciatura inédita). Universidad Nacional, Heredia, Costa Rica.

Valverde, S., \& Boza, J. (1998). Inducción al desove en hembras del pargo mancha, Lutjanus guttatus (Steindachner, 1869). Uniciencia, 15-16, 65-69.

Vega, L. A., \& Marín, C. (2014). Evaluation of Escherichia coli concentrations in Crassostrea gigas and seawater in two oyster growing areas in the Gulf of Nicoya, Costa Rica. Revista Ciencias Marinas y Costeras, 6, 155-166. http://dx.doi.org/10.15359/revmar.6.11

Vega, L., Marín, C., Pacheco, O., \& Zúñiga, G. (2013). Preliminary assessment of small scale bacterial depuration of Crassostrea gigas and Anadara spp., Gulf of Nicoya, Costa Rica. Revista Ciencias Marinas y Costeras, 5, 107-117.

Zúñiga, G. (2002). Evaluación del crecimiento de la ostra del Pacífico Crassostrea gigas (Pelecypoda: Ostreidae) en la finca camaronera "Langostinos del Pacífico", en Quepos y en algunos sitios del Golfo de Nicoya (Tesis de licenciatura inédita). Universidad Nacional, Heredia, Costa Rica.

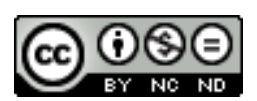

Avances biotecnológicos sobre maricultura en Costa Rica. Una revisión de la investigación desarrollada por la Escuela de Ciencias Biológicas de la Universidad Nacional (Rebeca Rojas-Alfaro y otros) por Revista Uniciencia se encuentra bajo una Licencia CreativeCommons Atribución-NoComercial-SinDerivadas 3.0 Unported. 